\title{
Sulfur-Doped and Bio-Resin-Derived Hard Carbon@rGo Composites as Sustainable Anodes for Lithium-Ion Batteries
}

\author{
Qinyuan Huang, Jinbo Hu*, Shujing Wen, Xiang Zhang, Gonggang Liu, Shanshan Chang* \\ and Yuan Liu
}

College of Materials Science and Engineering, Central South University of Forestry and Technology, Changsha, China

OPEN ACCESS

Edited by:

Weijie Li,

University of Wollongong, Australia

Reviewed by:

Xing-Long $W u$,

Northeast Normal University, China

Xianhong Rui,

Guangdong University of

Technology, China

*Correspondence:

Jinbo Hu

hjb1999@hotmail.com

Shanshan Chang

changelxy@hotmail.com

Specialty section

This article was submitted to

Electrochemistry,

a section of the journal

Frontiers in Chemistry

Received: 14 January 2020

Accepted: 13 March 2020

Published: 02 April 2020

Citation:

Huang Q, Hu J, Wen S, Zhang $X$,

Liu G, Chang S and Liu Y (2020)

Sulfur-Doped and Bio-Resin-Derived

Hard Carbon@rGO Composites as

Sustainable Anodes for Lithium-lon

Batteries. Front. Chem. 8:241

doi: 10.3389/fchem.2020.00241
Hard carbon derived from fossil products is widely used as anode material for lithium-ion batteries. However, there are still several main shortcomings such as high cost, and poor rate performance, which restrict its wide application. Then tremendous efforts have been devoted to developing biomaterials in the battery applications. Recently, especially agricultural and industrial by-products have attracted much attention due to the electric double-layer capacitors. Herein, we report the sulfur-doped hard carbon (SHC) materials from the tannin-furanic resins (TF-Resin) of the derived agricultural by-products, followed by enveloping $\mathrm{rGO}$ on its surface through the hexadecyl trimethyl ammonium bromide. SHC provides sites for the storage of lithium, while the rGO layers can offer a highly conductive matrix to achieve good contact between particles and promote the diffusion and transport of ions and electrons. As a result, the SHC@rGO shows excellent lithium storage performance with initial discharge capacity around $746 \mathrm{mAh} \mathrm{g}^{-1}$ at a current density of $50 \mathrm{~mA} \mathrm{~g}^{-1}$, and shows superb stability keeping capacity retention of $91.9 \%$ after 200 cycles. Moreover, even at a high current density of 2,000 mAg ${ }^{-1}$, SHC@rGO still delivers a specific capacity of $188 \mathrm{mAg}^{-1}$. These desired promising properties are active to the implement in the possible practical application.

Keywords: bio-resin-derived hard carbon, tannin-furanic resins, sulfur-doped, rGO, lithium-ion battery

\section{INTRODUCTION}

Nowadays, lithium-ion batteries (LIBs) have been widely used with energy storage systems and portable digital devices because of their long cycle stability and high energy density (Etacheri et al., 2011; Wang et al., 2014). With a high demand for LIBs, the preparation of low-cost, environmentally friendly, and high-performance anode materials has been substantially researched in recent years (Liao et al., 2012). Various carbonaceous materials, such as graphitic carbon (Funabiki et al., 1999; Song et al., 2011), amorphous carbon (hard carbon) (Zhang et al., 2015, 2016, 2017) and soft carbon (Chae et al., 2014; Huang et al., 2018; Wang et al., 2018)), nanostructured carbon (graphene) (Raccichini et al., 2016; Ferguson et al., 2017), and carbon nanotubes (Liu et al., 2018; Yuan et al., 2018) have been widely investigated for their LIBs applications. Among all the anode materials for LIBs, graphitic material is the most commercially unitized because of its low cost, low potential $\left(\approx 0.2 \mathrm{~V}\right.$ vs. $\left.\mathrm{Li} / \mathrm{Li}^{+}\right)$, and optimal electrical conductivity (Han et al., 2015). Nevertheless, graphite anode is far from meeting the demands for high energy/power density as a result of its limited capacity $\left(372 \mathrm{mAh} \mathrm{g}^{-1}\right)$ and inferior rate performance (Ge et al., 2018). 
Alternatively, majority of researchers have taken notice of hard carbon because of its high specific capacity (740 mAh $\mathrm{g}^{-1}$ ) (Fey and Chen, 2001). As well-known, the precursors for preparing hard carbon are mainly petrochemical raw materials, such as a phenolic resin (Liu et al., 1996), high molecular polymers (Piotrowska et al., 2013), asphalt (Larcher et al., 1999; Mochida et al., 2001), etc. These raw materials are non-renewable substances, and the price is volatile because of fluctuations in international oil prices. Also, carbonization of these products often causes environmental issue such as the release of formaldehyde or other toxic carcinogens. Fortunately, biomass is a rich and renewable potentially green material. A large amount of biomass with little or no economic value can be used as a cheap and effective source of carbon precursors to produce materials that are environmentally and economically of high added value. Most natural resources have been reported including bamboo chopsticks (Jiang et al., 2014), wood (Zhang et al., 2019), soybean (Xu et al., 2015), wheat flour (Lim et al., 2017), corn cob (Liu et al., 2016), peanut shells (Ding et al., 2015), cherry stones (Arrebola et al., 2010), silk (Hou et al., 2015), coconut oil (Gaddam et al., 2016), and mangrove (Liu et al., 2010), were widely used as electrodes for energy storage applications. However, the lower conductivity of hard carbon than that of graphitizable carbons is another defect, which results in poor rate performance. In order to tackle these problems, great efforts have been made to adjust the surface structure of hard carbon, such as the construction of hybrid anodes (Guo et al., 2008), thermal carbon coatings (Ohzawa et al., 2005; Lee et al., 2007), and vacuum and oxidation treatments (Fujimoto et al., 2010; Liu et al., 2010). Although these surface modification methods may improve the coulombic efficiency of hard carbon, their rate performance and cyclability are far from satisfactory. Moreover, the further modification optimization of hard carbon materials is quite meaningful for practical application.

Corn cob and wood bark are abandoned agricultural byproducts and are generally burnt, which not only leads to air pollution but also wastes resources. To date, tanninfuranic resins have received great attention because of tannin and furfural alcohol, which are extracted from wood bark and corn cob (Tondi and Pizzi, 2009; Meikleham and Pizzi, 2010). Thus far, the synthesis of resins without formaldehyde has been an attractive focus (Tondi et al., 2009; Li et al., 2013). With the development of technology, possible applications of the natural tannin-furanic resin were recently presented (Tondi et al., 2016). It is active that the epoxy novolac resin as the hard carbon source and coated rGO for LIBs have shown the outstanding stability and rate capability due to via constructing the conductive network (Zhang et al., 2015). Herein, we report the synthesis of SHC materials derived from the biomass TF-resin with the carbonization method and enveloped with the rGO on its surface through the hexadecyl trimethyl ammonium bromide. SHC provides active sites for the storage of lithium, whereas the rGO layer can offer a highly conductive matrix to achieve contact between particles and promote the diffusion and transport of ions and electrons. As a result, the SHC@rGO shows excellent lithium storage performance with an initial discharge capacity of approximately $746 \mathrm{mAh}$ $\mathrm{g}^{-1}$ at a current density of $50 \mathrm{~mA} \mathrm{~g}^{-1}$, and shows superb stability keeping a capacity retention of $91.9 \%$ after 200 cycles. Furthermore, even at a high current density of $2,000 \mathrm{~mA} \mathrm{~g}^{-1}$, SHC@rGO still delivers specific capacity of $188 \mathrm{~mA} \mathrm{~g}^{-1}$. This study compares the following petrochemical raw materials: (i) tannins and furfuryl alcohol raw materials, which have the advantages of environmental friendliness, low-cost, and renewability; (ii) the full biomass-derived hard carbon, which has optimal electrochemical properties; and (iii) it provides a reference for the application of the full biomass resin materials in energy storage materials.

\section{EXPERIMENTAL SECTION}

Tannin extract $(60 \mathrm{~g})$, furfuryl alcohol $(180 \mathrm{~g}), 100 \mathrm{~mL}$ deionized water, and $8 \mathrm{~mL}$ p-toluene sulfonic acid (65 wt\%), which acted as the hardener, were mixed at room temperature. The precursor was treated at $60^{\circ} \mathrm{C}$ for $1 \mathrm{~h}$ in a rotary evaporator and then cured in the drying box at 60,100 , and $150^{\circ} \mathrm{C}$ for 2,1 , and $24 \mathrm{~h}$, respectively. The cured precursor was heated at $500^{\circ} \mathrm{C}$ for $1 \mathrm{~h}$ with $3^{\circ} \mathrm{C} \mathrm{min}^{-1}$ and then ground to powders after cooling under the argon atmosphere. Finally, the sample was heated at $1,000^{\circ} \mathrm{C}$ under the argon flow for $1 \mathrm{~h}$ with a ramp rate of $10^{\circ} \mathrm{C} \mathrm{min}^{-1}$ to prepare the SHC.

SHC $(2 \mathrm{~g})$ and GO $(0.4 \mathrm{~g})$ were sonicated for $0.5 \mathrm{~h}$ in $120 \mathrm{~mL}$ (20 wt\%) ethanol to disperse GO sheets and SHC powders. The $0.1 \mathrm{~g}$ hexadecyl trimethyl ammonium bromide was dissolved in the resulting suspended liquid through magnetic stirring for $5 \mathrm{~h}$ at room temperature. The suspension was vacuum filtered, dried in air at $100^{\circ} \mathrm{C}$, and then heated to $1,000^{\circ} \mathrm{C}$ under argon flow for $1 \mathrm{~h}$ at a heating rate of $10^{\circ} \mathrm{C} \mathrm{min}^{-1}$ in a vacuum tube furnace to prepare the SHC@rGO.

\section{MATERIAL CHARACTERIZATION}

The morphologies of the sample were investigated with a scanning electron microscope (SEM). The morphology and microstructure of the SHC@rGO sample were characterized by transmission electron microscopy (TEM F20) and scanning transmission electron microscopy equipped with an energy dispersive spectrometer (EDS). The X-ray photoelectron spectra (XPS) were obtained by a Thermo Fisher Scientific ESCALAB 250Xi spectrometer. X-ray diffraction (XRD) was recorded on an XD-2X X-ray diffractometer with $\mathrm{Cu}$ $\mathrm{K} \alpha$ radiation $(\lambda=1.5406 \mathrm{~A}, 30 \mathrm{KV}, 20 \mathrm{~mA})$ at the scan rate of $8^{\circ} \mathrm{C} \mathrm{min}^{-1}$. Raman spectra were collected using a Raman spectrometer (Alpha300-R) with $532 \mathrm{~nm}$. Nitrogen adsorption and desorption isotherms were determined through nitrogen physisorption on a Micro Active for an ASAP 2460 analyzer. Comprehensive thermal analysis (TG-DSC) was obtained using a SETARAM AETSYS-24 comprehensive thermal analysis instrument, starting from room temperature to $1,200^{\circ} \mathrm{C}$ at a heating rate of $10^{\circ} \mathrm{C} \mathrm{min}^{-1}$ under an $\mathrm{N}_{2}$ gas atmosphere. 


\section{ELECTROCHEMICAL TESTING}

Electrochemical measurements were performed using a 2016 coin-type battery using lithium metal as the anode. The working electrode was composed of active materials (SHC and SHC@rGO), the conductivity material (super P), and the binder (PVDF) in a weight ratio of 8:1:1 dissolved in N-methyl pyrrolidinone. The slurry was then spread evenly on the copper foil and dried in a vacuum oven at $120^{\circ} \mathrm{C}$ for $12 \mathrm{~h}$. After drying, the electrode was cut into a disc with a diameter of $12 \mathrm{~mm}$. The carrying mass of SHC@rGO on the anode is 0.55$0.66 \mathrm{mg} \cdot \mathrm{cm}^{-2}$. The mass loads of rGO in SHC@rGO is $0.11-$ $0.13 \mathrm{mg} \cdot \mathrm{cm}^{-2}$. A solution of $1 \mathrm{M} \mathrm{LiPF}_{6}$ in ethylene carbonate, diethyl carbonate, and dimethyl carbonate (1:1:1 by volume ratio) was used as an electrolyte, and a polypropylene film was used as a separator. The cycling and rate performance of SHC and SHC@rGO were tested on a land battery test system at $25^{\circ} \mathrm{C}$. Cyclic voltammetry (CV) and electrochemical impedance spectroscopy (EIS) measurements were performed on an Ivium electrochemical workstation.

\section{RESULTS AND DISCUSSION}

Figure 1 shows the schematic illustration for the synthesis of SHC and SHC@rGO materials derived from the biomass TF-Resin. Firstly, TF-Resin precursors were obtained a simple solidify method (see Experimental Section for detail). Subsequently, the obtained TF-Resin precursors were calcinated under Ar atmosphere to prepare SHC. Finally, the SHC@rGO was obtained after enveloped with the $\mathrm{rGO}$ on its surface through the hexadecyl trimethyl ammonium bromide and assembled into a 2016 Coin battery.

Figure 2 shows the SEM images of SHC and SHC@rGO. It can be seen that SHC sample presents irregular morphology and the surface is non-porosity and glossy (Figures $\mathbf{2 A , B}$ ). The SHC@rGO sample consists of irregular HC particles and lamellar reduced graphene oxide ( $\mathrm{rGO}$ ) with a wrinkled surface (Figures 1C,D). which is help construct the conductive network and guaranteeing fast electron conduction.

The microstructure of SHC@rGO composites was further studied by TEM and high-resolution TEM (HRTEM). As presented in Figure 3A, the SHC is partially wrapped by corrugated graphene nanosheets. It can be known that winkled rGO is well bonded to SHC particles due to van der Waals force between graphene sheets and SHC, which is beneficial to transport electrons and lithium ions. HRTEM image of SHC@rGO sample taken on the edges of rGO is shown in Figure 3B. The rGO consists of several stacked layers and its thickness is $<5 \mathrm{~nm}$. Graphene coating can effectively promote the transmission rate of electrons and ions and maintain the integrity of the conductive network, which contribute to improved electrochemical performance. The EDS mapping of $\mathrm{SHC}$ is shown in Figure 3C. The EDS element mapping of $\mathrm{C}$ and $\mathrm{S}$ confirms that the $\mathrm{S}$ element is evenly distributed throughout the hard carbon.

To determine the doping level and bonding configuration of S, we performed the XPS analyzation of SHC@rGO. As presented in Figure 4A, the predominant peaks at 165.0, 228.0, 285.0, 402.0 , and $532.0 \mathrm{eV}$ are assigned to $\mathrm{S} 2 \mathrm{p}, \mathrm{S} \mathrm{2s}, \mathrm{C} 1 \mathrm{~s}, \mathrm{~N} 1 \mathrm{~s}$, and $\mathrm{O} 1 \mathrm{~s}$, respectively. The $\mathrm{S} 2 \mathrm{p}$ spectra (Figure $4 \mathrm{~B}$ ) showed three
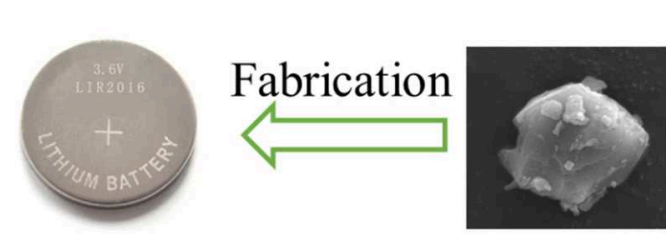

Lithium battery
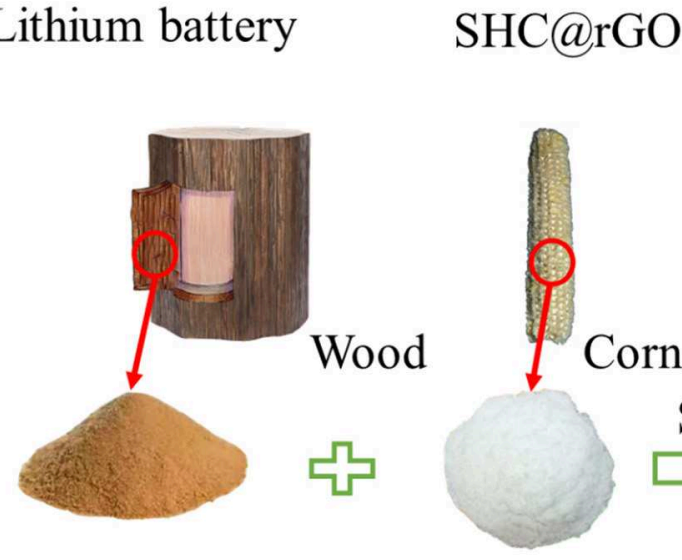

Tannin
Furfuryl alcohol
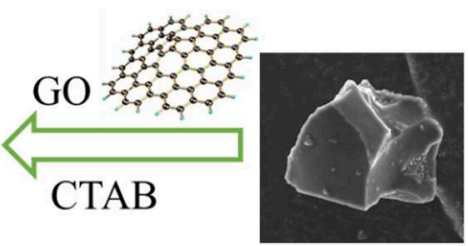

$\mathrm{SHC}$

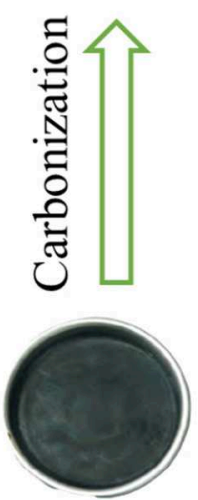

Solidify

TF-Resin

FIGURE 1 | Schematic illustration of the preparation of SHC and SHC@rGO. 

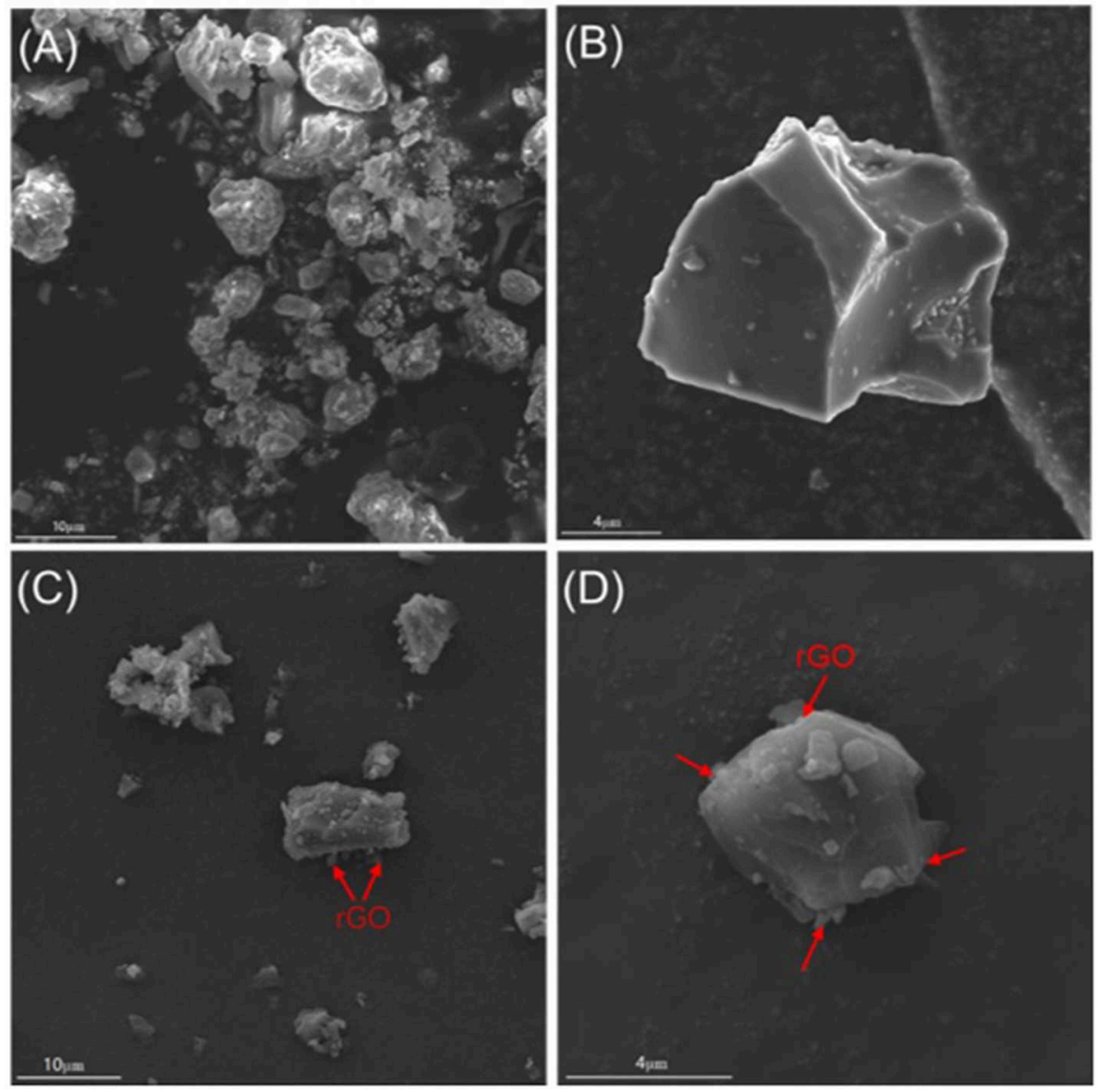

FIGURE 2 | SEM images of SHC (A,B) and SHC@rGO (C,D) composites.

peaks at 164.1, 165.3, and $169.2 \mathrm{eV}$. The former two peaks are assigned to the thiophene-S groups (-C-S-C-), and the third peak corresponds to the $-\mathrm{C}-\mathrm{SOx}$ groups $(X=2-4)$ (Choi et al., 2011; Kiani et al., 2019). The XPS results indicate that the $\mathrm{S}$ atom has been chemically bonded to the SHC.

The XRD patterns of the SHC and SHC@rGO samples are presented in Figure 5A. It can be seen that the XRD patterns of SHC and SHC@rGO are both composed of wide diffraction peaks at $22^{\circ}-26^{\circ}$ and $44^{\circ}-45^{\circ} 2 \theta$ corresponding to (002) and (100), respectively, which are typical of carbonaceous materials with an amorphous-like structures (Inagaki et al., 2001). The $D_{002}$ values of SHC and SHC@rGO are summarized in Table S1. SHC@rGO $(0.351 \mathrm{~nm})$ exhibits smaller average interlayer distance than SHC $(0.380 \mathrm{~nm})$, which is induced by rGO addition, because of the graphene oxide is reduced at high temperature to form a layered structure with decreasing interlayer spacing.

Figure 5B shows the Raman spectra of SHC and SHC@rGO. The characteristic peaks of the two samples are similar. The D-bands peak at about $1,340 \mathrm{~cm}^{-1}$ and the G-bands peak at about $1,590 \mathrm{~cm}^{-1}$. Peak D-bands and G-bands represent $\mathrm{sp}^{3}$ hybridization and $\mathrm{sp}^{2}$ hybridization, respectively. The ratio of the integrated areas of the two peaks can manifest the degree of order of carbon materials. The $I_{D} / I_{G}$ of the SHC and SHC@rGO is 1.13 and 1.08, respectively (Ferrari and Robertson, 2000). It was shown that the carbon atoms in $\mathrm{sp}^{2}$ hybridization increased after graphene was added, the structure order and graphitization degree of the materials increased. This result was consistent with the results of XRD.

The nitrogen adsorption-desorption isotherm test $(77 \mathrm{~K})$ is an important tool of characterizing the specific surface area and pore volume of porous materials. Figure $5 \mathrm{C}$ shows the $\mathrm{N}_{2}$ adsorptiondesorption curve and the pore size distribution based on the BJH (Barrett-Joiner-Halenda) model of SHC and SHC@rGO. It can be seen that the $\mathrm{N}_{2}$ adsorption isotherm of SHC and SHC@rGO belong to the III type isotherm. The calculated pore size distributions from the adsorption branches using the density functional theory (DFT) (inset of Figure 5C) model for SHC and SHC@rGO, suggest the characteristics of mesoporosity and microporosity. The specific surface area $S_{\mathrm{BET}}$ and pore volume data are listed in Table S1. It can be seen from the table that the BET specific surface area increases from $11.21 \mathrm{~m}^{2} \mathrm{~g}^{-1}$ of SHC to $21.02 \mathrm{~m}^{2} \mathrm{~g}^{-1}$ of SHC@rGO, the pores volume remains basically comparative. For these two samples, the mesopore volume accounts for more than $90 \%$ of the total pore volume. 


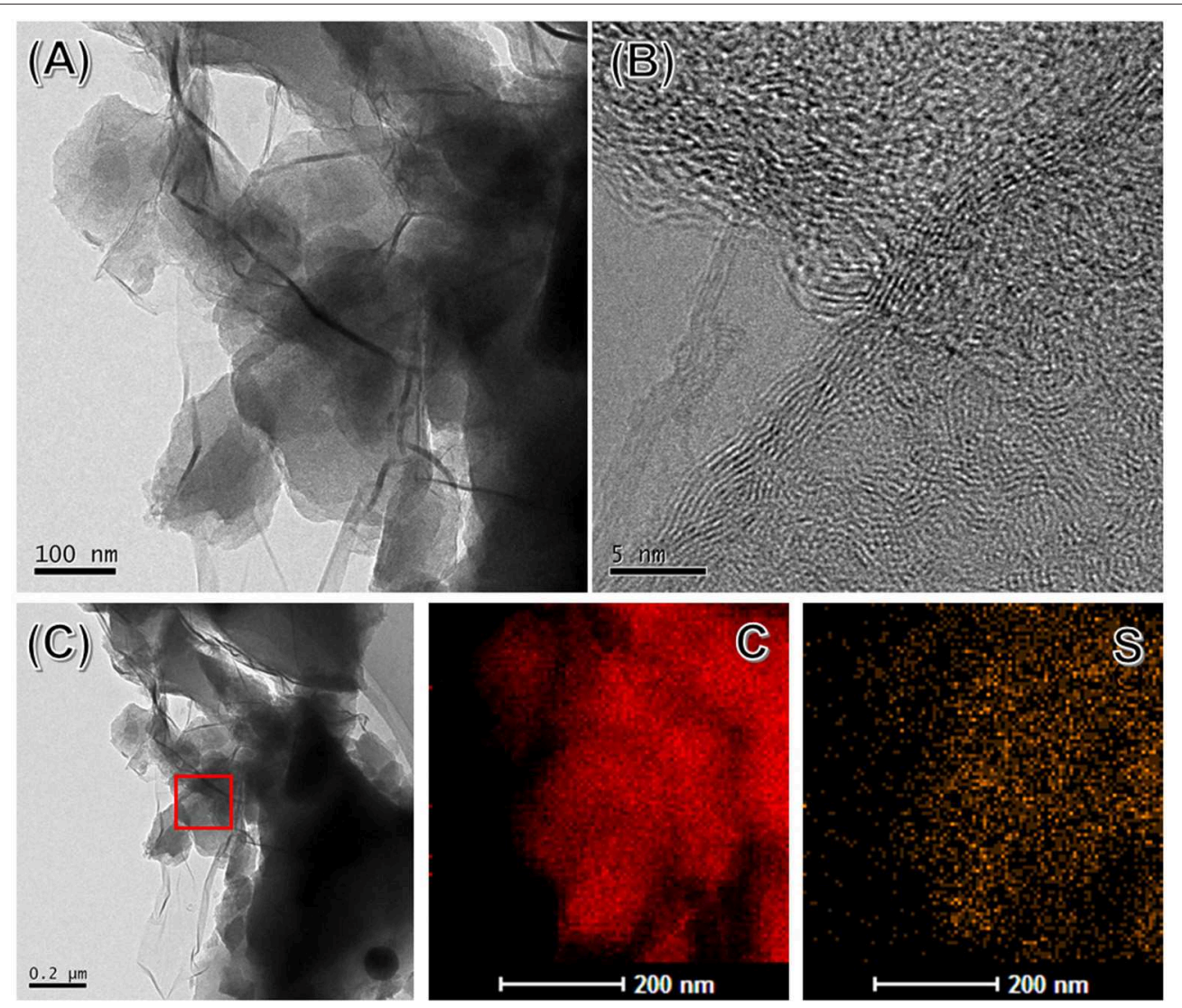

FIGURE 3 | A TEM (A), HRTEM image (B), and EDS elemental mapping (C) of SHC@rGO.
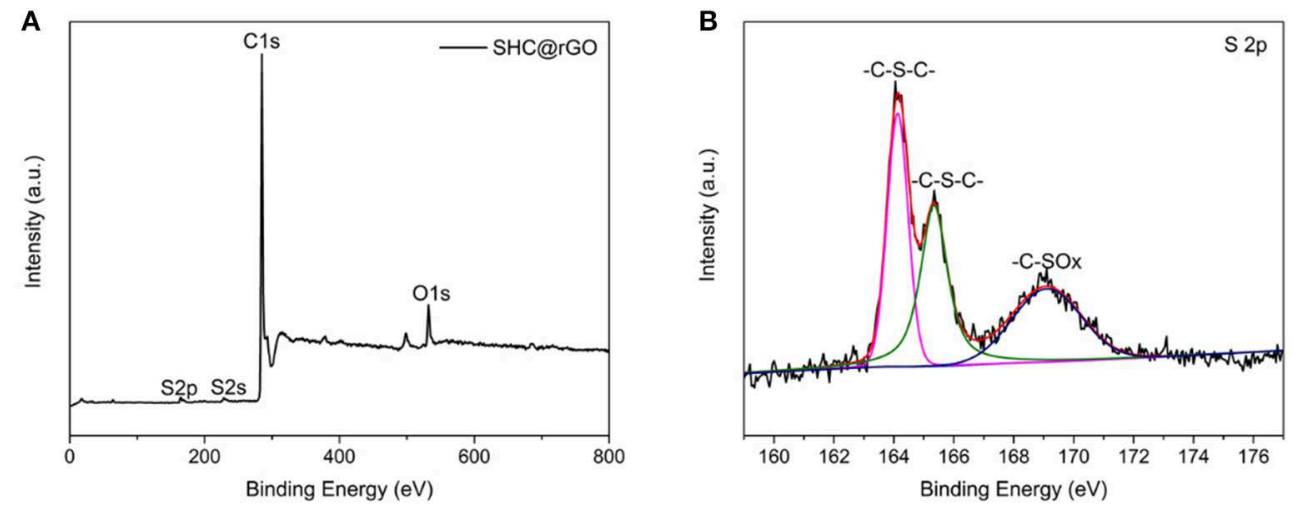

FIGURE 4 | XPS spectra of SHC@rGO composite and survey spectrum (A) and S 2p spectra (B).

Figure 5D shows the TG/DSC curves for GO and SHC@rGO. The TG/DSC of pure SHC curve is not shown because it has no heat loss and exotherm. It can be seen that GO has a distinct exothermic peak at $190^{\circ} \mathrm{C}$ with significant thermal weight loss, which is caused by the volatilization of a large number of water molecules adsorbed on GO. The thermogravimetric curve of SHC@rGO showed a slight decrease due to the small proportion of GO in the SHC@rGO mixture. When the temperature increases from 254 to $388^{\circ} \mathrm{C}$, the DSC curve of GO shows a taro peak with a slight thermal weight loss, indicating a moderate exothermic reaction, which is ascribed to escape of the large number of $\mathrm{H}$ atoms in GO. When the temperature is at 781 and $1,000^{\circ} \mathrm{C}$, there is a significant endothermic peak with obvious thermal weight loss, the TG curve still slightly decreases, which is caused by the large amount of $\mathrm{O}$ atoms in $\mathrm{GO}$ continuously escaping at high temperature. It also shows that GO can be 
A

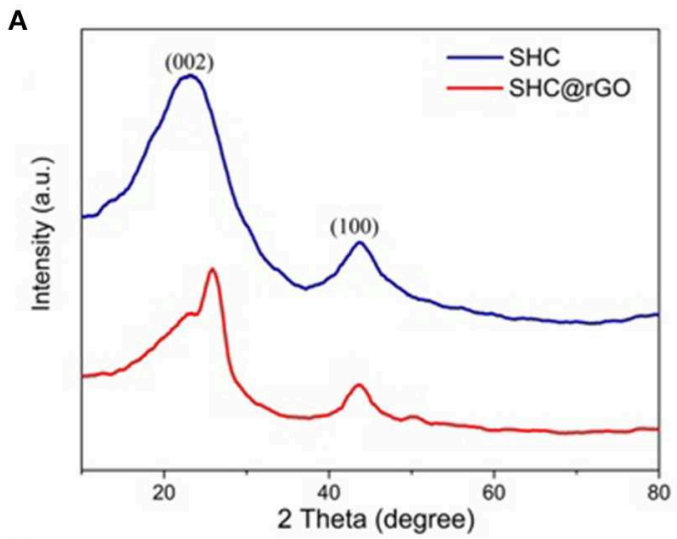

C

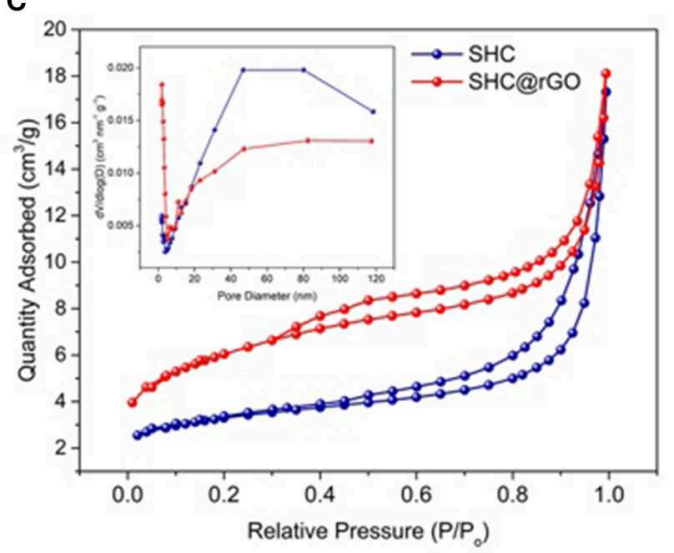

B
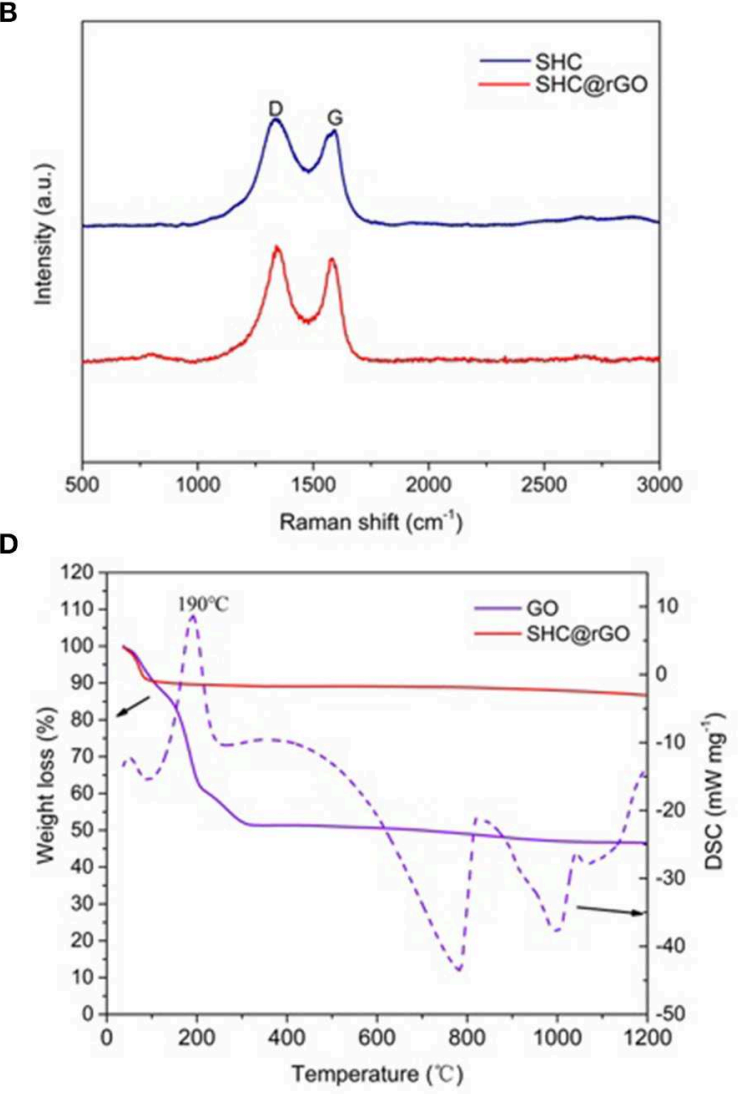

FIGURE 5 | (A) XRD patterns of SHC and SHC@rGO, (B) Raman spectra of SHC and SHC@rGO, (C) calculated pore size distribution and nitrogen adsorption isotherms (insert C) for SHC and SHC@rGO, and (D) TGA curve of the decomposition of SHC and SHC@rGO composite (Atmosphere: $\mathrm{N}_{2}$, heating rate: $10^{\circ} \mathrm{C}$ min ${ }^{-1}$ ).

reduced at high temperatures. When the pyrolysis temperature is $1,200^{\circ} \mathrm{C}$, the residual mass ratio of GO and SHC@rGO are 46.6 and $86.6 \%$, respectively. Assuming that the mass of SHC does not change, the theoretical calculation shows that the residual mass ratio of SHC@rGO is $88.3 \%$, which is basically consistent with the experimental results. It indicates that at high temperatures, most of the $\mathrm{H}$ and $\mathrm{O}$ atoms in the $\mathrm{GO}$ are removed.

The electrochemical profiles of SHC and SHC@rGO are characterized by coin half cells, which are composed of SHC or SHC@rGO as an anode, a lithium metal as counter electrodes, electrolyte, and a Celgard 2,400 separator. Figure 6 shows the initial two CV curves of SHC and SHC@rGO between 0 and $3 \mathrm{~V}$ at a sweep rate of $0.1 \mathrm{mV} \mathrm{s}^{-1}$. It can be seen that cathode reduction peaks appear at $0.70-0.75 \mathrm{~V}$ in the first cycle and disappear in the subsequent cycle (Figure 6B). These reduction peaks suggest that the solid electrolyte interphase (SEI) layer being formed on the surface of the carbon materials during the first lithium intercalation (Buqa et al., 2006). Cathodic current peaks reach $0.21 \mathrm{~V}$ because of irreversible side reactions of lithium with absorbed species or surface functional groups, a weak reduction peak was found in two samples during the first discharge process but disappeared in the second cycle (Figure 6B), which contributes to part of capacity loss leading to large irreversible capacity (Zhang et al., 2014). In addition, the anodization peak potential of SHC@rGO was lower than that of pure SHC, indicating that when graphene is added to the hard carbon material, lithium-ions are more easily deintercalated in the hard carbon material during the charge/discharge process, which will be beneficial to improve its electrochemical performance. Therefore, the graphene in SHC@rGO not only provides electronic conductivity but also improves the reversible migration ability of lithium-ions in materials.

The initial galvanostatic discharge/charge curves of SHC and SHC@rGO at a current density of $50 \mathrm{~mA} \mathrm{~g}^{-1}$ are shown in Figure 7A. The charging and discharging processes correspond to the lithium removal and insertion process, respectively. It can be seen that the shape of the first charge-discharge curves of SHC and SHC@rGO is analogical, indicating that the lithium intercalation/deintercalation mechanism of SHC has not been changed after modification by wrapped GO. It can be seen that the specific capacity of SHC and SHC@rGO for the initial discharge capacities are 605 and $746 \mathrm{mAh} \mathrm{g}^{-1}$, and the charge capacities are 321 and $486 \mathrm{mAh} \mathrm{g}^{-1}$, respectively. The first irreversible specific capacity of SHC and SHC@rGO are 284 and $260 \mathrm{mAhg}^{-1}$, and the initial Coulomb efficiency (ICE) of SHC and SHC@rGO are 53.1\% and 65.1\%, respectively. 

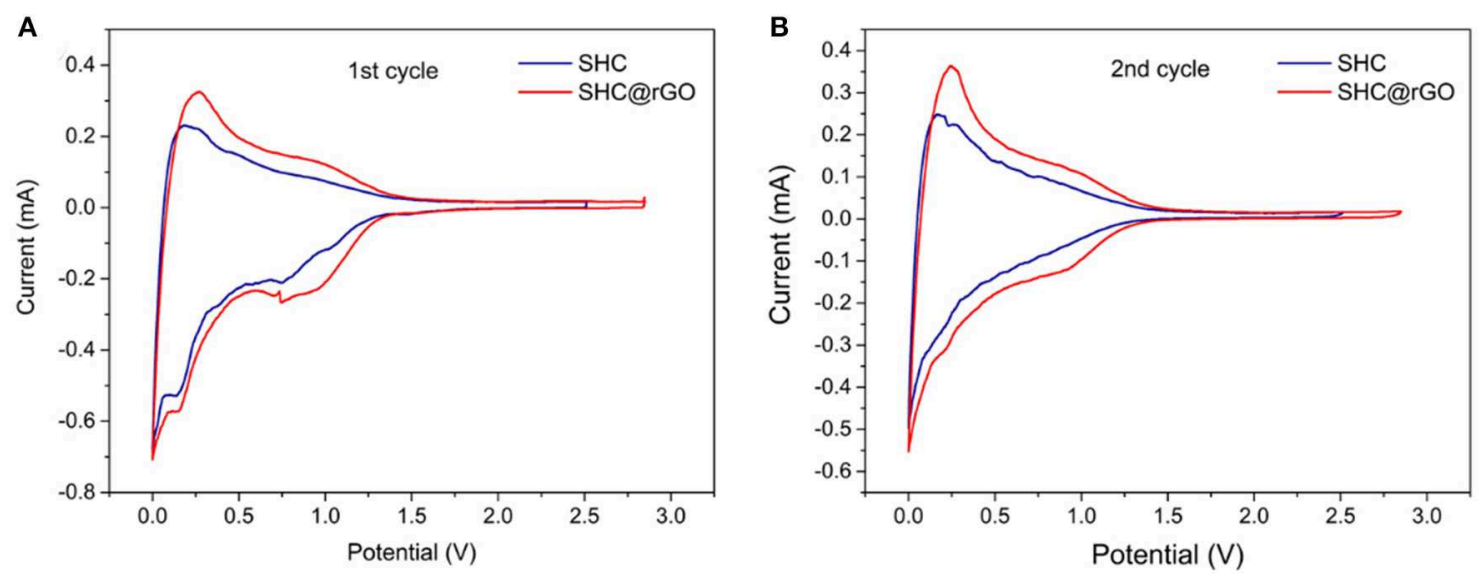

FIGURE 6 | (A) CV curves of SHC and SHC@rGO in the first cycle and (B) CV curves of SHC and SHC@rGO in the second cycle.
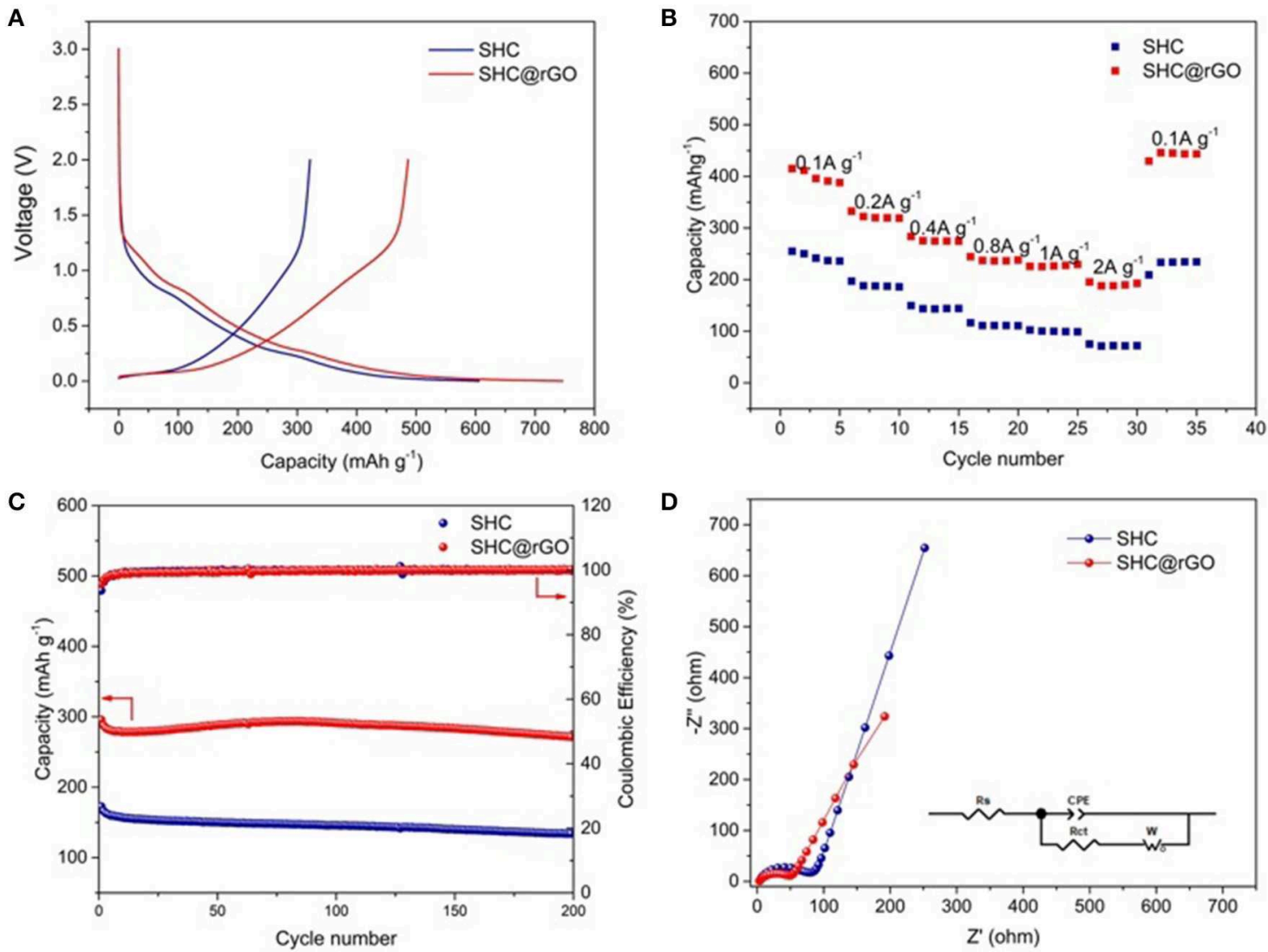

FIGURE 7 | (A) Charge and discharge curves of SHC and SHC@rGO at a current density of $50 \mathrm{~mA} \mathrm{~g}^{-1}$ between 0 and $3 \mathrm{~V}$, (B) ratio performance patterns of SHC and SHC@rGO with the charge/discharge current densities in the $100-2000 \mathrm{~mA} \mathrm{~g}^{-1}$ range, (C) cycle stability curves of SHC and SHC@rGO at $400 \mathrm{~mA} \mathrm{~g}^{-1}$, and (D) Nyquist plots of SHC and SHC@rGO at the potential of $0.1 \mathrm{~V} \mathrm{vs.} \mathrm{Li/Li+} \mathrm{over} \mathrm{the} \mathrm{frequency} \mathrm{range} \mathrm{from} 100 \mathrm{kHz}$ to $0.01 \mathrm{~Hz}$.

Unfortunately, the pure SHC electrodes show low ICE at the same current density, similar to previous reports about hard carbon material (Zhang et al., 2015). The battery of the low ICE is probably attributed to the following two major reasons.
Primarily, the decomposition of electrolyte during the initial charge/discharge process has significantly displayed in the battery formulation of SHC derived the resin. Secondly, the formation of an irreversible solid electrolyte interface (SEI) on the surface of 
anode could also lead to a low ICE (Liu et al., 2016), consistent with the CV results. Nevertheless, it is active that the ICE can be improved from 53.1 to $65.1 \%$ owing to the presence of rGO in SHC@rGO. The ICE improvement of SHC@rGO was ascribed to the crucial rGO, which can construct the conductive network and promote the transport of $\mathrm{Li}^{+}$ions in the electrolyte. Therefore, the SHC would be progressively modified in the prospective battery.

The rate performance of SHC and SHC@rGO prepared at different current densities are shown in Figure 7B. The rate performance of SHC@rGO is superior to that of SHC under different current densities from $0.1 \mathrm{~A} \mathrm{~g}^{-1}\left(410 \mathrm{mAh} \mathrm{g}^{-1}\right)$ to $2 \mathrm{~A} \mathrm{~g}^{-1}\left(188 \mathrm{mAh} \mathrm{g}^{-1}\right)$. When the current rate decreased from $2 \mathrm{~A} \mathrm{~g}^{-1}$ to $0.1 \mathrm{~A} \mathrm{~g}^{-1}$ after the 25 th rate cycle corresponding to discharge capacity of $444.7 \mathrm{mAh}^{-1}$. As for SHC, a much lower rate capacity was delivered at varied current densities. Both the capacity of SHC and SHC@rGO increased when the current density returned to $0.1 \mathrm{~A} \mathrm{~g}^{-1}$, this phenomenon can be confirmed by the electrolyte infiltration and electrode material activation during different current charge/discharge processes. Table S2 lists TF-resin prepared in this work vs. other hard carbons derived from epoxy novolac resin (Zhang et al., 2014), pitch (Kim et al., 2016), and polyphenylene sulfide (Luo et al., 2018), which demonstrates that the SHC derived from TF-resin deliver better discharge capacity and rate performance.

Cycle stability of SHC and SHC@rGO was estimated at the current density of $400 \mathrm{~mA} \mathrm{~g}^{-1}$ (Figure 7C). It can be inferred that from this cyclic performance, SHC and SHC@rGO delivered 134.1 and $271.8 \mathrm{mAh} \mathrm{g}^{-1}$ capacity after 200 cycles with the Coulombic efficiency near $100 \%$, keeping capacity retention of 77.7 and 91.9\%, respectively. Therefore, the SHC@rGO hybrid anode shows obviously higher cycling stability and reversible capacity, which are S-doping hard carbon provides active sites for the storage of lithium, while the rGO layers offer a highly conductive matrix and high contact area can lead to effective contact with the electrolyte into the electrode and quickly transport ions into the deeper parts of the SHC@rGO particles and graphite layer. A comparison of hard carbon prepared in this work with other biomass-derived carbons is shown in Table S3, which demonstrates that the prepared hard carbon delivers better rate capabilities and cycling performance.

The EIS measurements of SHC and SHC@rGO were tested (Figure 5D). The plots consist of a semicircle in the highfrequency region and a straight line in the low-frequency region. the straight line and the semicircle corresponding to Warburg diffusion impedance (W) and charge transfer resistance (Rct), respectively (Pan et al., 2018). Based on equivalent electric circuit, the Rct of SHC and SHC@rGO are 65.34 and 50.49 $\Omega$, respectively. The lower Rct of SHC@rGO was attributed to the

\section{REFERENCES}

Arrebola, J. C., Caballero, A., Hernán, L., Morales, J., Olivares-Marín, M, and Gómez-Serrano, V. (2010). Improving the performance of biomass-derived carbons in li-ion batteries by controlling the lithium presence of conductive rGO able to constructing the conductive network and facilitate the transport of $\mathrm{Li}^{+}$ions, resulting in a superior electrochemical performance.

\section{CONCLUSION}

In summary, biomass TF resin-derived SHC and SHC@rGO composite material was prepared as the anode material for lithium-ion battery. It is demonstrated that the rGO networks are enveloped on SHC constructing the desired microstructure. Compared with the biomass-derived pure SHC, the SHC@rGO composites show enhanced conductivity offered by rGO networks and large $\mathrm{Li}$ ion storage sites supported by SHC. As a consequence, SHC@rGO composite exhibits a superior electrochemical rate performance and reversible capacity. Based on this study, a new biomass derived hard carbon composites with superior electrochemical properties is reported, which is promising for low-cost and eco-friendly anode material of LIBs.

\section{DATA AVAILABILITY STATEMENT}

All datasets generated for this study are included in the article/Supplementary Material.

\section{AUTHOR CONTRIBUTIONS}

JH, XZ, SC, and YL: conceived and designed the experiments. $\mathrm{QH}, \mathrm{SW}$, and $\mathrm{JH}$ : performed the experiment. SC, JH, and $\mathrm{XZ}$ : supervised the work. QH, JH, and YL: wrote the paper. JH, GL, and XZ: revised the paper. JH and YL: contributed equally. All authors reviewed and approved the final manuscript.

\section{FUNDING}

The research was funded by the National Key Research and Development Program of China (2017YFB1303901; to JH) and the Scientific research project of Hunan Provincial Department of Education (19A505; to JH).

\section{ACKNOWLEDGMENTS}

The authors thank Priof. Xiaobo JI from Central South University in China for the analysis.

\section{SUPPLEMENTARY MATERIAL}

The Supplementary Material for this article can be found online at: https://www.frontiersin.org/articles/10.3389/fchem. 2020.00241/full\#supplementary-material

insertion process. J. Electrochem. Soc. 157, A791-A797. doi: 10.1149/1.34 25728

Buqa, H., Würsig, A., Vetter, J., Spahr, M. E., Krumeich, F., and Novák, P. (2006). SEI film formation on highly crystalline graphitic materials in lithium-ion batteries. J. Power Sour. 153, 385-390. doi: 10.1016/j.jpowsour.2005.05.036 
Chae, J. E., Annaka, K., Hong, K., Lee, S.-I., Munakata, H., Kim, S.-S., et al. (2014). Electrochemical characterization of phosphorous-doped soft carbon using single particle for lithium battery anode. Electrochim. Acta 130, 60-65. doi: 10.1016/j.electacta.2014.03.009

Choi, C. H., Park, S. H., and Woo, S. I. (2011). Heteroatom doped carbons prepared by the pyrolysis of bio-derived amino acids as highly active catalysts for oxygen electro-reduction reactions. Green Chem. 13, 406-412. doi: 10.1039/C0GC00384K

Ding, J., Wang, H. L., Li, Z., Cui, K., Karpuzov, D., Tan, X. H., et al. (2015). Peanut shell hybrid sodium ion capacitor with extreme energy-power rivals lithium ion capacitors. Energy Environ. Sci. 8, 941-955. doi: 10.1039/C4EE02986K

Etacheri, V., Marom, R., Elazari, R., Salitra, G., and Aurbach, D. (2011). Challenges in the development of advanced Li-ion batteries: a review. Energy Environ. Sci. 4, 3243-3262. doi: 10.1039/clee01598b

Ferguson, D., Searles, D. J., and Hankel, M. (2017). Biphenylene and phagraphene as lithium ion battery anode materials. ACS Appl. Mater. Interfaces 9, 20577-20584. doi: 10.1021/acsami.7b04170

Ferrari, A. C., and Robertson, J. (2000). Interpretation of Raman spectra of disordered and amorphous carbon. Phys. Rev. B 61, 14095-14107. doi: 10.1103/PhysRevB.61.14095

Fey, T. K., and Chen, C. L. (2001). High-capacity carbons for lithiumion batteries prepared from rice husk. J. Power Sour. 97, 47-51. doi: 10.1016/S0378-7753(01)00504-3

Fujimoto, H., Tokumitsu, K., Mabuchi, A., Chinnasamy, N., and Kasuh, T. (2010). The anode performance of the hard carbon for the lithium ion battery derived from the oxygen-containing aromatic precursors. J. Power Sour. 195, 7452-7456. doi: 10.1016/j.jpowsour.2010.05.041

Funabiki, A., Inaba, M., Abe, T., and Ogumi, Z. (1999). Nucleation and phase-boundary movement upon stage transformation in lithiumgraphite intercalation compounds. Electrochim. Acta 45, 865-871. doi: 10.1016/S0013-4686(99)00290-X

Gaddam, R. R., Yang, D., Narayan, R., Raju, K., Kumar, N. A., and Zhao, X. S. (2016). Biomass derived carbon nanoparticle as anodes for high performance sodium and lithium ion batteries. Nano Energy 26, 346-352. doi: 10.1016/j.nanoen.2016.05.047

Ge, C., Fan, Z., Zhang, J., Qiao, Y., Wang, J., and Ling, L. (2018). Novel hard carbon/graphite composites synthesized by a facile in situ anchoring method as high-performance anodes for lithium-ion batteries. RSC Adv. 8, 34682-34689. doi: 10.1039/C8RA07170E

Guo, B., Shu, J., Tang, K., Bai, Y., Wang, Z., and Chen, L. (2008). Nano-Sn/hard carbon composite anode material with high-initial coulombic efficiency. J. Power Sour. 177, 205-210. doi: 10.1016/j.jpowsour.2007.11.003

Han, Y.-J., Kim, J., Yeo, J.-S., An, J. C., Hong, I.-P., Nakabayashi, K., et al. (2015). Coating of graphite anode with coal tar pitch as an effective precursor for enhancing the rate performance in Li-ion batteries: effects of composition and softening points of coal tar pitch. Carbon 94, 432-438. doi: 10.1016/j.carbon.2015.07.030

Hou, J., Cao, C., Idrees, F., and Ma, X. (2015). Hierarchical porous nitrogen-doped carbon nanosheets derived from silk for ultrahigh-capacity battery anodes and supercapacitors. ACS Nano 9, 2556-2564. doi: 10.1021/nn506394r

Huang, F., Zhao, Q., Yang, J., Zhang, H., Huo, W., and Xu, F. (2018). Spherical mesophase soft carbon materials with micro-nano composite structure and their applications in lithium-ion batteries. Energy Sour. Part A: Recovery Utilization Environ. Effects 40, 1-6. doi: 10.1080/15567036.2018.1486897

Inagaki, M., Fujita, K., Takeuchi, Y., Oshida, K., Iwata, H., and Konno, H. (2001). Formation of graphite crystals at $1000-1200^{\circ} \mathrm{C}$ from mixtures of vinyl polymers with metal oxides. Carbon 39, 921-929. doi: 10.1016/S0008-6223(00) 00210-4

Jiang, J., Zhu, J. H., Ai, W., Fan, Z. X., Shen, X. N., and Zou, C. J. (2014). Evolution of disposable bamboo chopsticks into uniform carbon fibers: a smart strategy to fabricate sustainable anodes for Li-ion batteries. Energy Environ. Sci. 7, 2670-2679. doi: 10.1039/C4EE00602J

Kiani, M., Zhang, J., Chen, J., Luo, Y., Chen, Y., Fan, J., et al. (2019). Facile synthesis of magnesium ferrite nanoparticles supported on nitrogen and sulfur co-doped carbon black as an efficient electrocatalyst for oxygen reduction reaction. J. Nanoparticle Res. 21. doi: 10.1007/s11051-019-4539-9

Kim, B.-H., Kim, J.-H., Kim, J.-G., Bae, M.-J., Im, J. S., Lee, C. W., et al. (2016). Electrochemical and structural properties of lithium battery anode materials by using a molecular weight controlled pitch derived from petroleum residue. $J$. Ind. Eng. Chem. 41, 1-9. doi: 10.1016/j.jiec.2016.07.006

Larcher, D., Mudalige, C., Gharghouri, M., and Dahn, J. R. (1999). Electrochemical insertion of $\mathrm{Li}$ and irreversibility in disordered carbons prepared from oxygen and sulfur-containing pitches. Electrochim. Acta 44, 4069-4072. doi: 10.1016/S0013-4686(99)00167-X

Lee, J.-H., Lee, H.-Y., Oh, S.-M., Lee, S.-J., Lee, K.-Y., and Lee, S.-M. (2007). Effect of carbon coating on electrochemical performance of hard carbons as anode materials for lithium-ion batteries. J. Power Sour. 166, 250-254. doi: 10.1016/j.jpowsour.2006.12.078

Li, X., Nicollin, A., Pizzi, A., Zhou, X., Sauget, A., and Delmotte, L. (2013). Natural tannin-furanic thermosetting moulding plastics. RSC Adv. 3, 17732-17740. doi: $10.1039 / \mathrm{c} 3 \mathrm{ra} 43095 \mathrm{~b}$

Liao, X., Ji, Y., and Gao, L. (2012). Electrochemical study on lithium iron phosphate/hard carbon lithium-ion batteries. J. Solid State Electrochem. 16, 423-428. doi: 10.1007/s10008-011-1387-7

Lim, D. G., Kim, K., Razdan, M., Diaz, R., Osswald, S., and Pol, V. G. (2017) Lithium storage in structurally tunable carbon anode derived from sustainable source. Carbon 121, 134-142. doi: 10.1016/j.carbon.2017.05.079

Liu, D.-S., Liu, D.-H., Hou, B.-H., Wang, Y.-Y., Guo, J.-Z., Ning, Q.-L., et al. (2018). 1D porous $\mathrm{MnO} @ \mathrm{~N}$-doped carbon nanotubes with improved Li-storage properties as advanced anode material for lithium-ion batteries. Electrochim. Acta 264, 292-300. doi: 10.1016/j.electacta.2018.01.129

Liu, P., Li, Y. M., Hu, Y. S., Li, H., Chen, L. Q., and Huang, X. J. (2016). A waste biomass derived hard carbon as a high-performance anode material for sodium-ion batteries. J. Mater. Chem. A 4, 13046-13052. doi: 10.1039/C6TA04877C

Liu, T., Luo, R., Qiao, W., Yoon, S.-H., and Mochida, I. (2010). Microstructure of carbon derived from mangrove charcoal and its application in Li-ion batteries. Electrochim. Acta 55, 1696-1700. doi: 10.1016/j.electacta.2009.10.051

Liu, Y., Xue, J. S., Zheng, T., and Dahn, J. R. (1996). Mechanism of lithium insertion in hard carbons prepared by pyrolysis of epoxy resins. Carbon 34, 193-200. doi: 10.1016/0008-6223(96)00177-7

Luo, D., Chen, M., Xu, J., Yin, X., Wu, J., Chen, S., et al. (2018). Polyphenylene sulfide nonwoven-based composite separator with superior heat-resistance and flame retardancy for high power lithium ion battery. Compos. Sci. Technol. 157, 119-125. doi: 10.1016/j.compscitech.2018.01.023

Meikleham, N. E., and Pizzi, A. (2010). Acid- and alkali-catalyzed tannin-based rigid foams. J. Appl. Polym. Sci. 53, 1547-1556. doi: 10.1002/app.1994.070531117

Mochida, I., Ku, C.-H., and Korai, Y. (2001). Anodic performance and insertion mechanism of hard carbons prepared from synthetic isotropic pitches. Carbon 39, 399-410. doi: 10.1016/S0008-6223(00)00137-8

Ohzawa, Y., Yamanaka, Y., Naga, K., and Nakajima, T. (2005). Pyrocarboncoating on powdery hard-carbon using chemical vapor infiltration and its electrochemical characteristics. J. Power Sour. 146, 125-128. doi: 10.1016/j.jpowsour.2005.03.009

Pan, Q., Zheng, F., Wu, Y., Ou, X., Yang, C., and Xiong, X. (2018). MoS2-covered SnS nanosheets as anode material for lithium-ion batteries with high capacity and long cycle life. J. Mater. Chem. A 6, 592-598. doi: 10.1039/C7TA08346G

Piotrowska, A., Kierzek, K., Rutkowski, P., and Machnikowski, J. (2013). Properties and lithium insertion behavior of hard carbons produced by pyrolysis of various polymers at $1000^{\circ}$ C. J. Anal. Appl. Pyrolysis 102, 1-6. doi: 10.1016/j.jaap.2013.04.011

Raccichini, R., Varzi, A., Chakravadhanula, V. S. K., Kübel, C., and Passerini, S. (2016). Boosting the power performance of multilayer graphene as lithium-ion battery anode via unconventional doping with in-situ formed Fe nanoparticles. Sci. Rep. 6:23585. doi: 10.1038/srep23585

Song, M.-K., Park, S., Alamgir, F. M., Cho, J., and Liu, M. (2011) Nanostructured electrodes for lithium-ion and lithium-air batteries: the latest developments, challenges, and perspectives. Mater. Sci. Eng. R Rep. 72, 203-252. doi: 10.1016/j.mser.2011.06.001

Tondi, G., Link, M., Kolbitsch, C., Lesacher, R., and Petutschnigg, A. (2016). Pilot plant up-scaling of tannin foams. Ind. Crops Prod. 79, 211-218. doi: 10.1016/j.indcrop.2015.11.013

Tondi, G., and Pizzi, A. (2009). Tannin-based rigid foams: characterization and modification. Ind. Crops Prod. 29, 356-363. doi: 10.1016/j.indcrop.2008. 07.003 
Tondi, G., Zhao, W., Pizzi, A., Du, G., Fierro, V., and Celzard, A. (2009). Tannin-based rigid foams: a survey of chemical and physical properties. Bioresour. Technol. 100, 5162-5169. doi: 10.1016/j.biortech.2009. 05.055

Wang, D., Zhou, J., Li, Z., Li, J., Hou, L., and Gao, F. (2018). Uniformly expanded interlayer distance to enhance the rate performance of soft carbon for lithium-ion batteries. Ionics 25, 1531-1539. doi: 10.1007/s11581-0182646-8

Wang, F., Li, W., Hou, M., Li, C., Wang, Y., and Xia, Y.-Y. (2014). Sandwichlike Cr2O3-graphite intercalation composites as high-stable anode materials for lithium-ion batteries. J. Mater. Chem. A, 3:1703. doi: 10.1039/C4TA 05072J

Xu, G., Han, J., Ding, B., Nie, P., Pan, J., and Dou, H. (2015). Biomassderived porous carbon materials with sulfur and nitrogen dual-doping for energy storage. Green Chem. 17, 1668-1674. doi: 10.1039/C4GC0 2185A

Yuan, W., Qiu, Z., Chen, Y., Zhao, B., Liu, M., and Tang, Y. (2018). A binderfree composite anode composed of $\mathrm{CuO}$ nanosheets and multi-wall carbon nanotubes for high-performance lithium-ion batteries. Electrochim. Acta 267, 150-160. doi: 10.1016/j.electacta.2018.02.081

Zhang, X., Fan, C., Li, L., Zhang, W., Zeng, W., He, X., et al. (2014). Hard carbon wrapped in graphene networks as lithium ion battery anode. Electrochim. Acta 149, 94-100. doi: 10.1016/j.electacta.2014.10.094

Zhang, X., Fan, C. L., and Han, S. C. (2017). Improving the initial coulombic efficiency of hard carbon-based anode for rechargeable batteries with high energy density. J. Mater. Sci. 52, 10418-10430. doi: 10.1007/s10853-0171206-3

Zhang, X., Han, S. C., Fan, C. L., Li, L. F., and Zhang, W. H. (2015). Hard carbon enveloped with graphene networks as lithium ion battery anode. Mater. Lett. 138, 259-261. doi: 10.1016/j.matlet.2014.10.023

Zhang, X., Han, S. C., Xiao, P. G., Fan, C. L., and Zhang, W. H. (2016). Thermal reduction of graphene oxide mixed with hard carbon and their high performance as lithium ion battery anode. Carbon 100, 600-607. doi: 10.1016/j.carbon.2016.01.033

Zhang, X., Hu, J., Chen, X., Zhang, M., Huang, Q., Du, X., et al. (2019). Microtubular carbon fibers derived from bamboo and wood as sustainable anodes for lithium and sodium ion batteries. J. Porous Mater. 26, 1821-1830. doi: 10.1007/s10934-019-00781-3

Conflict of Interest: The authors declare that the research was conducted in the absence of any commercial or financial relationships that could be construed as a potential conflict of interest.

Copyright (c) 2020 Huang, Hu, Wen, Zhang, Liu, Chang and Liu. This is an openaccess article distributed under the terms of the Creative Commons Attribution License (CC BY). The use, distribution or reproduction in other forums is permitted, provided the original author(s) and the copyright owner(s) are credited and that the original publication in this journal is cited, in accordance with accepted academic practice. No use, distribution or reproduction is permitted which does not comply with these terms. 